\title{
On subset seeds for protein alignment
}

\author{
Mikhail Roytberg, Anna Gambin, Laurent Noé, Sławomir Lasota, Eugenia \\ Furletova, Ewa Szczurek, Gregory Kucherov
}

A preliminary version of this paper appeared in the proceedings of the ALBIO'08 workshop (Vienna, Austria July 7-9, 2008)

Mikhail Roytberg and Eugenia Furletova are with the Institute of Mathematical Problems in Biology, Pushchino, Moscow Region, 142290, Russia, e-mail: mroytberg@mail.ru, furletova@impb.psn.ru

Anna Gambin and Sławomir Lasota are with the Institute of Informatics, Warsaw University, Banacha 2, 02-097, Poland, \{aniag|S.Lasota\}@mimuw.edu.pl

Ewa Szczurek is with the Max Planck Institute for Molecular Genetics, Computational Molecular Biology, Ihnestr. 73, 14195 Berlin, Germany, ewa.szczurek@molgen.mpg.de

Gregory Kucherov and Laurent Noé are with LIFL/CNRS/INRIA, Bât. M3, Campus Scientifique, 59655 Villeneuve d'Ascq Cédex, France, \{Gregory.Kucherov|Laurent.Noe\}@lifl.fr 


\begin{abstract}
We apply the concept of subset seeds proposed in [1] to similarity search in protein sequences. The main question studied is the design of efficient seed alphabets to construct seeds with optimal sensitivity/selectivity trade-offs. We propose several different design methods and use them to construct several alphabets. We then perform a comparative analysis of seeds built over those alphabets and compare them with the standard BLASTP seeding method [2], [3], as well as with the family of vector seeds proposed in [4]. While the formalism of subset seeds is less expressive (but less costly to implement) than the cumulative principle used in BLASTP and vector seeds, our seeds show a similar or even better performance than BLASTP on Bernoulli models of proteins compatible with the common BLOSUM62 matrix. Finally, we perform a large-scale benchmarking of our seeds against several main databases of protein alignments. Here again, the results show a comparable or better performance of our seeds vs. BLASTP.
\end{abstract}

\title{
Index Terms
}

protein sequences, protein databases, local alignment, similarity search, seeds, subset seeds, multiple seeds, seed alphabet, sensitivity, selectivity

\section{INTRODUCTION}

Similarity search in protein sequences is probably the most classical bioinformatics problem, and a commonly used algorithmic solution is implemented in the ubiquitous BLAST software [2], [3]. On the other hand, similarity search algorithms for nucleotide sequences (DNA, RNA) underwent several years ago a significant improvement due to the idea of spaced seeds and its various generalizations [5], [6], [7], [8], [9], [10]. This development, however, has little affected protein sequence comparison, although improving the speed/precision tradeoff for protein search would be of great value for numerous bioinformatics projects. Due to a bigger alphabet size, protein seeds are much shorter (typically 2-5 letters instead of 10-20 letters in the DNA case) and also letter identity is much less relevant in defining hits than in the DNA case. For these reasons, the spaced seeds technique might seem not to apply directly to protein sequence comparison.

Recall that BLAST applies quite different approaches to protein and DNA sequences to define a hit. In the DNA case, a hit is defined as a short pattern of identically matching nucleotides whereas in the protein case, a hit is defined through a cumulative contribution of a few amino acid matches (not necessarily identities) using a given scoring matrix. Defining a hit through an additive contribution of several positions is captured by a general formalism 
of vector seeds proposed in [11]. On the other hand, it has been understood [7], [12], [13], [14], [15] that using simultaneously a family of seeds instead of a single seed can further improve the sensitivity/selectivity ratio. Papers [4], [16] both propose solutions using a family of vector seeds to surpass the performance of BLAST.

However, using the principle of cumulative score over several adjacent positions has an algorithmic cost. Defining a hit through a pattern of exact letter matches allows for a direct hashing scheme, where each key of the query sequence is associated with a unique hash table entry pointing to the positions of the subject sequence (database) where the key can hit. Usually these positions are stored in consecutive memory cells within the hash table.

On the other hand, defining a hit through a cumulative contribution of several positions leads to an additional pre-computed table that stores, for each key, its neighborhood i.e., the list of subject keys (or corresponding hash table entries) with which it can form a hit. For example, in a standard BLASTP setting (Blosum62 scoring matrix with threshold 11 for cumulative score of three positions), the expectation, computed according to the Bernoulli sequence model, of the number of neighbors of a key is 19.34 , i.e. that many accesses to the hash table are required for each key. For four positions and threshold 18, as in the case of seeds from [4], a key hits expectedly 15.99 keys and this number grows up to 45.59 when the score threshold decreases to 16. This raises an obvious memory problem: for example, for key size 4 and score threshold 18, the total size of neighborhoods is 7609575 , and for key size 5 the neighborhood table may simply not fit into the memory. Another related implementation problem is cache usage: different keys of a neighborhood generally correspond to remote segments of the hash table and their processing gives rise to cache misses that cause additional latencies.

Those implementation issues may become a bottleneck in large-scale protein comparisons. Furthermore, solving these problems may be very helpful in different specific experimental setups, such as in mapping protein comparison algorithms to specialized computer architecture (see e.g. [17], [18]) where memory usage may be a crucial issue.

In [1], we proposed a new concept of subset seeds that can be viewed as an intermediate between ordinary spaced seeds and vector seeds: subset seeds allow one to distinguish between different types of mismatches (or matches) but still treat seed positions independently rather than cumulatively. Distinguishing different mismatches is not done by scoring them, but by extending the seed alphabet such that each seed letter specifies different sets of mismatches. For example, in the DNA case it is beneficial to distinguish between transition 
mutations ( $\mathrm{A} \leftrightarrow \mathrm{G}, \mathrm{C} \leftrightarrow \mathrm{T}$ ) and others (transversions) [19], [20]. This leads (at least in the case of transitive seed alphabets defined in this paper) to the possibility of using the direct hashing.

Since the protein alphabet is much larger than the one of DNA, subset seeds provide a very attractive seeding option for protein alignment. In this paper, we study the performance of subset seeds applied to protein sequences and compare it to existing seeding techniques of BLASTP and vector seeds.

Note again that subset seeds are less expressive than BLAST seeds or vector seeds in general, but in return, admit a more efficient implementation. Besides treating positions independently, subset seeds replace amino acid substitution scores by simply distinguishing different classes of mismatches. Therefore, another way to state the motivation of this work is to ask whether scores are really necessary at the seeding stage of protein alignment. We will show that with a reasonable level of precision the answer to this question is negative.

In the paradigm of subset seeds, each seed letter specifies a set of amino acid pairs matched by this letter. Therefore, a crucial question is the design of an appropriate seed alphabet, which is one of the main problems we study in this paper. In fine, the quality of an alphabet is determined by the quality of the best seeds that can be constructed over this alphabet. The latter is already a complex optimization problem that is usually solved in practice by heuristic methods. (For a formal analysis of seed design problem we refer to the recent paper [21] and references therein.) The problem of alphabet design studied in this paper presents an additional complexity as it introduces an additional dimension of the search space (set of possible alphabets), and additionally requires a study of selectivity/sensitivity dependencies rather than simply maximizing the sensitivity for a class of seeds with a given selectivity. In this paper we propose several heuristic methods that lead to the design of efficient seed alphabets and corresponding seeds.

The paper is organized as follows. In Section $\amalg$, we introduce some probabilistic notions we need to reason about seed efficiency. Section $\amalg$ introduces the first simple approach to design a seed alphabet, which, however, does not lead to so-called transitive seeds, useful in practice. Section IV presents three different approaches to designing transitive seed alphabets, based on a pre-defined (Section IV-A ) or newly designed (Section IV-B hierarchical clustering of amino acids, as well as on a non-hierarchical clustering (Section IV-C). Section $\mathrm{V}$ describes comparative experiments made with the designed seeds, obtained both on probabilistic models and on different protein data banks. 


\section{PRELIMINARIES}

Throughout the paper, we denote $\Sigma=\{\mathrm{A}, \mathrm{C}, \mathrm{D}, \mathrm{E}, \mathrm{F}, \mathrm{G}, \mathrm{H}, \mathrm{I}, \mathrm{K}, \mathrm{L}, \mathrm{M}, \mathrm{N}, \mathrm{P}, \mathrm{Q}, \mathrm{R}, \mathrm{S}, \mathrm{T}, \mathrm{V}, \mathrm{W}, \mathrm{Y}\}=$ $\left\{a_{i}\right\}_{i=1 . .20}$ the alphabet of amino acids.

In most general terms, a (subset) seed letter $\alpha$ is defined as any symmetric and reflexive binary relation on $\Sigma$. Let $\mathcal{B}$ be a seed alphabet, i.e. a collection of subset seed letters. Then a subset seed $\pi=\alpha_{1} \ldots \alpha_{k}$ is a word over $\mathcal{B}$, where $k$ is called the span of $\pi . \pi$ defines a symmetric and reflexive binary relation on words of $\Sigma^{k}$ (called keys): for $s_{1}, s_{2} \in \Sigma^{k}$, $s_{1} \sim_{\pi} s_{2}$ iff $\forall i \in[1 . . k]$, we have $\left\langle s_{1}[i], s_{2}[i]\right\rangle \in \alpha_{i}$. In this case, we say that seed $\alpha$ hits the pair $s_{1}, s_{2}$.

For practical reasons, we would like seed letters to define a transitive relation, in addition. This induces an equivalence relation on keys, which is very convenient and allows for an efficient indexing scheme (see Introduction). In this paper, we will be mainly interested in transitive seed letters, but we will also study the non-transitive case in order to see how restrictive the transitivity condition is.

The quality of a seed letter or of a seed is characterized by two main parameters: sensitivity and selectivity. They are defined through background and foreground probabilistic models of protein alignments. Foreground probabilities are assumed to represent the distribution of amino acids matches in proteins of interest, when two homologous proteins are aligned together. Background probabilities, on the other hand, represent the distribution of amino acid matches in random alignments, when two proteins are randomly aligned together.

In this paper, we restrict ourselves to Bernoulli models of proteins and protein alignments, although some of the results we will present can be extended to Markov models.

Assume that we are given background probabilities $\left\{b_{1}, \ldots, b_{20}\right\}$ of amino acids in protein sequences under interest. The background probability of a seed letter $\alpha$ is defined by $b(\alpha)=$ $\sum_{\left(a_{i}, a_{j}\right) \in \alpha} b_{i} b_{j}$. The selectivity of $\alpha$ is $1-b(\alpha)$ and the weight of $\alpha$ is defined by

$$
w(\alpha)=\frac{\log b(\alpha)}{\log b(\#)}
$$

where $\#=\{\langle a, a\rangle \mid a \in \Sigma\}$ is the "identity" seed letter. For a seed $\pi=\alpha_{1} \ldots \alpha_{k}$, the background probability of $\pi$ is $b(\pi)=\prod_{i=1}^{k} b\left(\alpha_{i}\right)$, the selectivity of $\pi$ is $1-b(\pi)$ and the weight of $\pi$ is $w(\pi)=\log _{b(\#)} b(\pi)=\sum_{i=1}^{k} w\left(\alpha_{i}\right)$. Note that the weight here generalizes the weight of classical spaced seeds [22] defined as the number of "identity" letters it contains.

Let $f_{i j}$ be the probability to see the pair $\left\langle a_{i}, a_{j}\right\rangle$ aligned in a target alignment. The foreground probability of a seed letter $\alpha$ is defined by $f(\alpha)=\sum_{\left(a_{i}, a_{j}\right) \in \alpha} f_{i j}$. The sensitivity 
of a seed $\pi$ is defined as the probability to hit a random target alignment 1 . Assume that target alignments are specified by a length $N$. Then the sensitivity of a seed $\pi=\alpha_{1} \ldots \alpha_{k}$ is the probability that a randomly drawn gapless alignment (i.e. string of pairs $\left\langle a_{i}, a_{j}\right\rangle$ ) of length $N$ contains a fragment of length $k$ which is matched by $\pi$. In [1] we proposed a general algorithm to efficiently compute the seed sensitivity for a broad class of target alignment models. This algorithm will be used in the experimental part of this work.

The general problem of seed design is to obtain seeds with good sensitivity/selectivity trade-off. Even within a fixed seed formalism, the quality of a seed is dependent on the chosen selectivity value. This is why we will always be interested in computing efficient seeds for a large range of selectivity levels.

\section{DOMINATING SEED LETTERS}

Our main question is how to choose seed letters that form good seeds? Intuitively, "good letters" are those that best distinguish foreground and background letter alignments.

For each letter $\alpha$, consider its foreground and background probabilities $f(\alpha)$ and $b(\alpha)$ respectively. Intuitively, we would like to have letters $\alpha$ with large $f(\alpha)$ and small $b(\alpha)$. A letter $\alpha$ is said to dominate a letter $\beta$ if $f(\alpha) \geq f(\beta)$ and $b(\alpha) \leq b(\beta)$. Observe that in this case, $\beta$ can be removed from consideration, as it can always be advantageously replaced by $\alpha$.

Consider all amino acid pairs $\left(a_{i}, a_{j}\right)$ ordered by descending likelihood ratio $f_{i j} / b_{i} b_{j}$. Consider the set of pairs $R(t)=\left\{\left(a_{i}, a_{j}\right) \mid f_{i j} / b_{i} b_{j}>t\right\}$. Then the following statement holds 2 .

Proposition 1: $R(t)$ cannot be dominated by any other letter.

Proof: Assume by contradiction that $R(t)$ is dominated by some letter $\alpha$, i.e. $f(\alpha) \geq$ $f(R(t))$ and $b(\alpha) \leq b(R(t))$. Consider $\beta=R(t) \backslash \alpha$ and $\gamma=\alpha \backslash R(t)$. Clearly, $f(\beta) \leq$ $f(\gamma)$ and $b(\beta) \geq b(\gamma)$. On the other hand, $\forall\left(a_{i}, a_{j}\right) \in \beta, f_{i j} / b_{i} b_{j}>t$ and $\forall\left(a_{i}, a_{j}\right) \in \gamma$, $f_{i j} / b_{i} b_{j} \leq t$. This implies that $f(\beta)=\sum_{\left(a_{i}, a_{j}\right) \in \beta} f_{i j}>t \sum_{\left(a_{i}, a_{j}\right) \in \beta} b_{i} b_{j}=t b(\beta)$ and similarly

\footnotetext{
${ }^{1}$ Note that our definitions of sensitivity and selectivity are not symmetric: sensitivity is defined with respect to the entire alignment and selectivity with respect to a single alignment position. These definitions capture better the intended parameters we want to measure. However, selectivity could also be defined with respect to the entire alignment. We could suggest the term specificity for this latter definition.

${ }^{2}$ It is interesting to point out the relationship to the Neyman-Pearson lemma which is a more general formulation of this statement.
} 


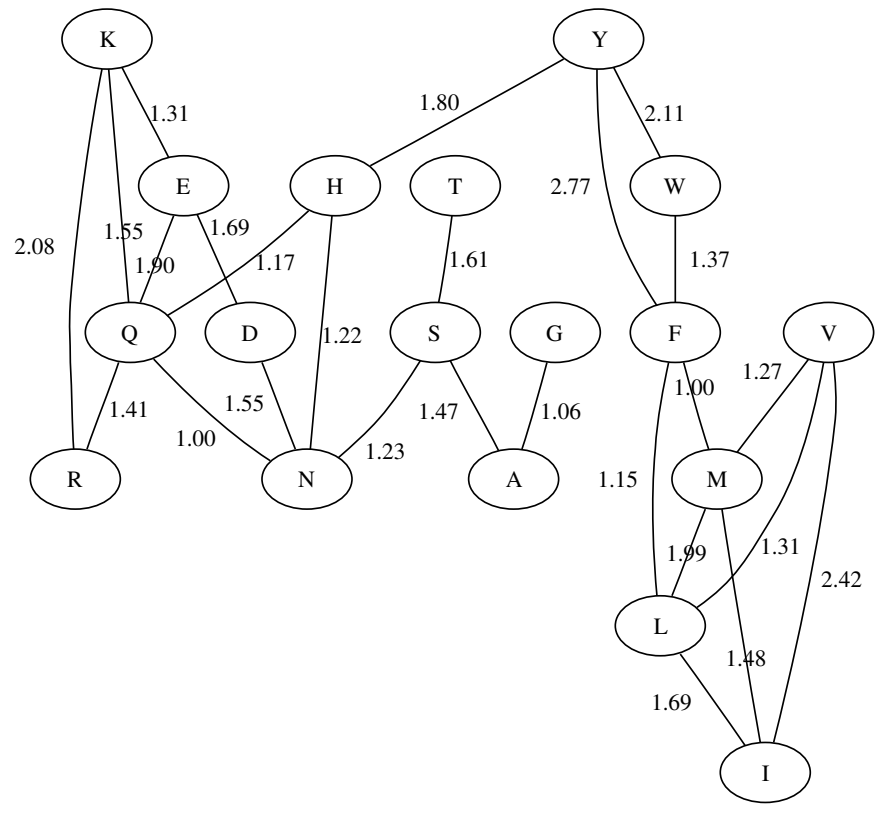

Fig. 1

AMINO ACID PAIRS FORMING LETTER $R(1)$ OF ALPHABET NON-TRANS I I I VE

$f(\gamma) \leq t b(\gamma)$. We then have $f(\beta)>t b(\beta) \geq t b(\gamma) \geq f(\gamma)$ which contradicts $f(\beta) \leq f(\gamma)$.

Proposition 1 suggests that letters $R(t)$ are good candidates to be included to the seed alphabet.

Resulting alphabet. We computed the likelihood ratio for all amino acid pairs, based on practical values of background and foreground probabilities computed in accordance with the BLOSUM62 matrix (see Section $\mathrm{V}$-A ). Not surprisingly, amino acid identities (pairs $\langle a, a\rangle$ ) have highest likelihood scores varying from 38.11 for tryptophan (W) down to 3.69 for valine (V).

Among non-identical pairs, only 25 have a score greater than 1 (Figure 1). A quick analysis shows that those do not form a transitive relation, and therefore $R(1)$ does not verify the transitivity requirement. This is also the case for other threshold values.

We analyzed a family of threshold letters $R(t)$ for $t$ ranging from 0 to 3 with step 0.05 . At the extremities of this interval, $R(0)$ is the "joker" letter admitting all amino acid pairs, and $R(3)$ is the letter corresponding to the exact match relation. Among all those letters, there are only 34 different ones. This alphabet of 34 letters (data not shown), denoted 
Non-transitive, will be used in the experimental part of the paper (Section $V$ ) in order to study how restrictive the requirement of transitive letters is, i.e. how much better are general seeds than those obtained with the restriction of transitivity.

\section{TRANSITIVE SEED ALPHABETS}

In the case of transitive seed alphabets, every letter $\alpha \in \mathcal{B}$ is a partition of the amino acid alphabet $\Sigma$. In other words, the binary relation associated with each letter (cf Section II) is an equivalence relation. Transitive alphabets represent the practical case when each amino acid is uniquely mapped to its equivalence class. This, in turn, allows for an efficient hashing scheme during the stage of seed search, when different entries of the hash table index nonintersecting subsets of keys.

In Sections IV-A and IV-B below, we explore transitive seed alphabets satisfying an additional "hierarchy condition": for any two seed letters $\alpha_{1}, \alpha_{2} \in \mathcal{B}$ corresponding to partitions $P_{\alpha_{1}}, P_{\alpha_{2}}$ respectively, one of $P_{\alpha_{1}}, P_{\alpha_{2}}$ is a refinement of the other. Formally,

$$
\text { for any } \alpha_{1}, \alpha_{2} \in \mathcal{B} \text {, either } \alpha_{1} \prec \alpha_{2} \text {, or } \alpha_{2} \prec \alpha_{1} \text {, }
$$

where $\alpha \prec \beta$ means that every set of $P_{\beta}$ is a subset of some set of $P_{\alpha}$.

The purpose of the above requirement is to define seed letters using a biologically significant hierarchical clustering of amino acids represented by a tree. In Section IV-A, we will use a pre-defined hierarchical clustering to design efficient seed alphabets. Then in Section IV-B, we construct our own clustering based on appropriate background and foreground models of amino acids distribution. Finally, in Section IV-C we lift condition (2) and study "nonhierarchical" seed alphabets.

\section{A. Transitive alphabets based on a pre-defined clustering}

Assume we have a biologically significant hierarchical clustering tree which is a rooted binary tree $T$ with 20 leaves labeled by amino acids. Such trees have been proposed in [23], [24], based on different similarity relations. The hierarchical tree derived from [23] is shown in Figure 2. The tree, obtained with a purely bioinformatics analysis, groups together amino acids with similar biochemical properties, such as hydrophobic amino acids $L, M, I, V$, hydrophobic aromatic amino acids $\mathrm{F}, \mathrm{Y}, \mathrm{W}$, amino acids with an alcohol group $\mathrm{S}, \mathrm{T}$, or charged/polar amino acids E, D, N, Q. A similar grouping has been obtained in [24].

A seed letter is defined here as a subset $\alpha$ of nodes of $T$ such that 


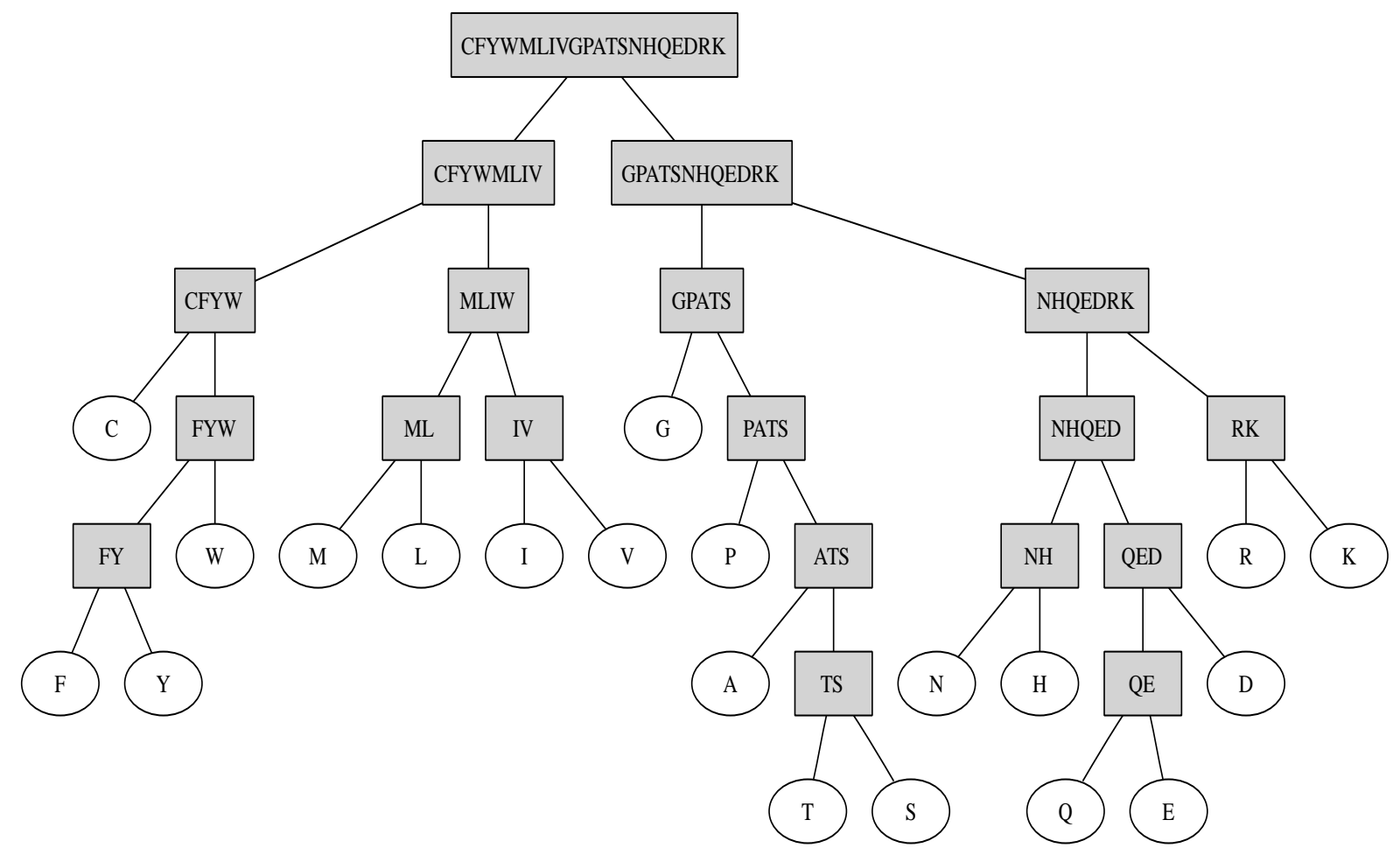

Fig. 2

HIERARCHICAL TREE DERIVED FROM [23]

(i) $\alpha$ contains all leaves,

(ii) for a node $v$, if $v \in \alpha$, then all descendants of $v$ belong to $\alpha$ too.

In other words, a seed letter can be thought of as a "horizontal cut" of the tree. Clearly, each letter induces a partition on the set of leaves (amino acids). For example, for the tree on Figure 2, a letter defined by the cut through nodes C, FYW, MLIV, G, P, ATS, NHQEDRK corresponds to the partition $\{\{C\},\{F Y W\},\{M L I V\},\{G\},\{P\},\{A T S\},\{$ NHQEDRK $\}$.

Seed letters are naturally ordered by inclusion. The smallest one is the "identity" seed letter \#, containing only the leaves. The largest one is the "joker" seed letter _, containing all the nodes of $T$. One particular seed letter is obtained by removing from - the root node. We denote it by @.

Observe that each seed letter $\alpha$ represents naturally an equivalence relation on $\Sigma: a_{i}$ and $a_{j}$ are related iff their common ancestor belongs to $\alpha$. It is identity relation in case of \# and full relation in case of _.

Following condition (2), a hierarchical seed alphabet is a family $\mathcal{B}$ of seed letters such 
that

$$
\text { for every } \alpha_{1}, \alpha_{2} \in \mathcal{B} \text {, either } \alpha_{1} \subseteq \alpha_{2} \text { or } \alpha_{2} \subseteq \alpha_{1} \text {. }
$$

Hence, in mathematical terms, a seed alphabet is a chain in the inclusion ordering of seed letters. Each hierarchical alphabet can be obtained by a series of refinements (set splittings) of its least refined letter.

Let us analyze what are the maximal seed alphabets wrt. inclusion. Clearly each maximal seed alphabet $\mathcal{B}$ always contains the smallest and the largest seed letters \# and _ . Interestingly, each maximal alphabet $\mathcal{B}$ contains also @, as @ is comparable (by inclusion) to any other seed letter.

It can be shown that under the above definitions, any maximal seed alphabet contains exactly 20 letters that can be obtained by a stepwise merging of two subtrees rooted at immediate descendants of some node $v$ into the subtree rooted at $v$. Therefore, since a binary tree with $n$ leaves contains $n-1$ internal nodes, a maximal seed alphabet contains precisely 20 letters and can be specified by a permutation of internal nodes in tree $T$.

Seed alphabets and constraint independence systems. It is interesting to observe that the set of seed alphabets forms a constrained independence system [25]. An independence system is a collection of subsets $\mathcal{I} \subseteq 2^{E}$ over a ground set $E$, called independent sets, such that (i) $\emptyset \in \mathcal{I}$, and (ii) if $X \in \mathcal{I}$ and $Y \subseteq X$, then $Y \in \mathcal{I}$. A maximal (w.r.t. inclusion) independent set is called a base.

Let $E$ be the set of all possible seed letters as defined earlier. Then alphabets verifying (3) form an independence system, where bases correspond to maximal seed alphabets. Moreover, seed alphabets verify two additional conditions of constrained independent system [25]: (iii) if $X, Y \in \mathcal{I}$ with $|Y|<|X|$, then there is an element $e \in E \backslash Y$ such that $Y \cup\{e\} \in \mathcal{I}$, and (iv) the cardinality of every minimal (w.r.t inclusion) set of $2^{E} \backslash \mathcal{I}$ is two.

The interest of this observation follows from results of [25] showing that some optimization problems on constrained independence systems can be solved efficiently by greedy algorithms. Assume we have a score function $s: E \rightarrow R$ that we extend additively to independent sets by $s(X)=\sum_{e \in X} s(e)$. For an independence system $\mathcal{I}$, we want to find a base $X \in \mathcal{I}$ with optimal (maximal or minimal) $s(X)$. For constrained independence systems, it was proved [25] that the greedy algorithm yields a base which is locally optimal, i.e. better than any neighbor base $Y=\left(X \backslash\left\{\alpha_{1}\right\}\right) \cup\left\{\alpha_{2}\right\}$ for some $\alpha_{1} \in X, \alpha_{2} \in E \backslash X$. Here, the greedy algorithm starts with the empty set and iteratively adds most optimal elements of $E$ as long 
as the current set remains independent. The absolute optimum is hard to compute in general, and the greedy solution is an approximation of it.

Assigning letter score. The above setting requires that each letter $\alpha$ is assigned a score that, intuitively, should measure the "usefulness" of $\alpha$ in a potential alphabet. Defining such a measure is a difficult question as there are too many potential alphabets and we can not check them all exhaustively. Therefore, we chose to consider only small alphabets $\mathcal{B}_{\alpha}$, containing $\alpha$ together with a few other letters that are always present in a good seed alphabet. Those letters are $\{-, @, \#\}$. The experiments reported in Section $\square$ use the alphabet $\mathcal{B}_{\alpha}=\{-, \alpha\}$.

Given $\mathcal{B}_{\alpha}$, we define the score of $\alpha$ as follows. We enumerate all seeds of a given span (typically, 5 or 6 ) over $\mathcal{B}_{\alpha}$, and compute the sensitivity and selectivity of each seed according

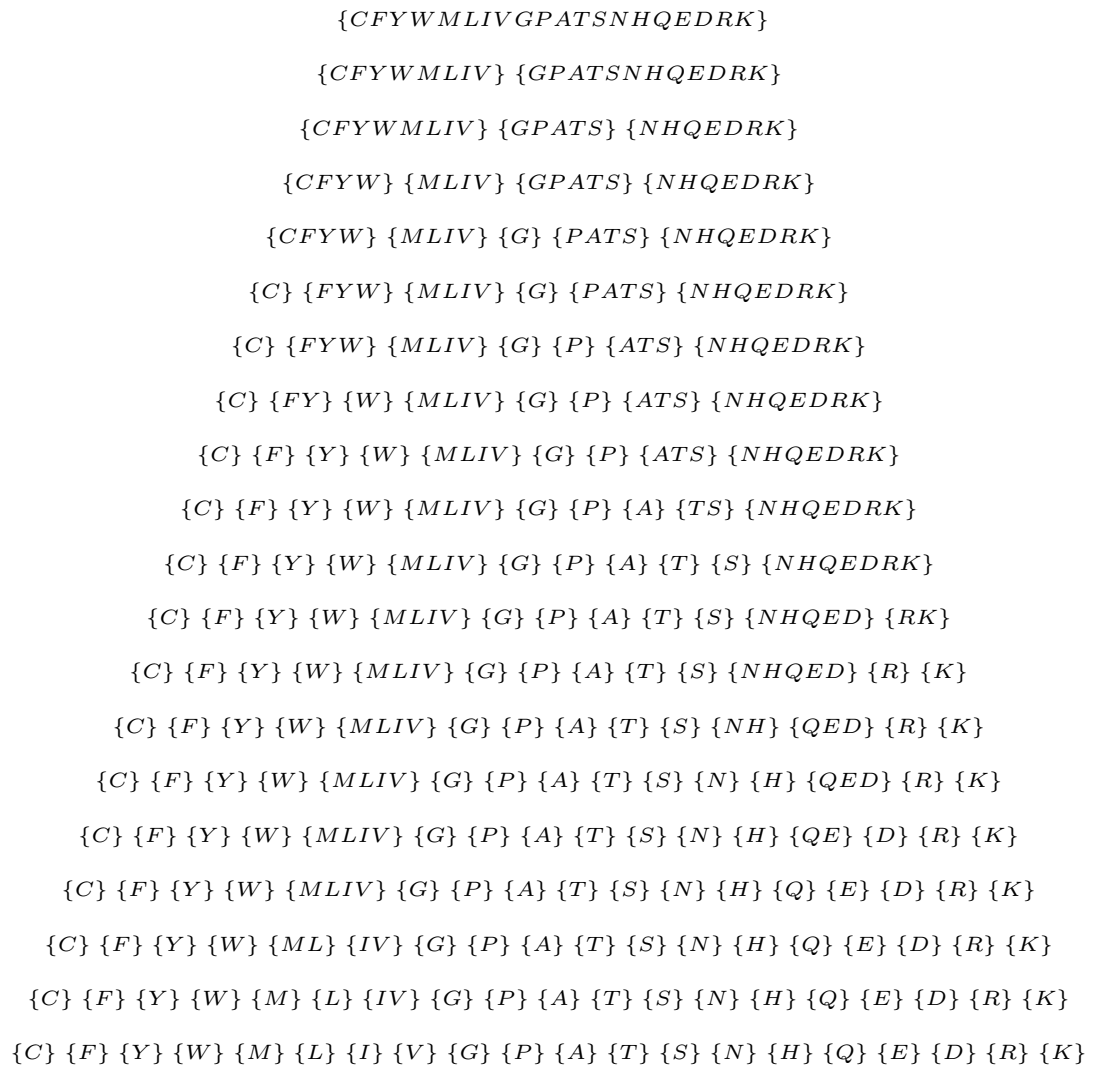

Fig. 3

Alphabet Trans it ive-Predefined Designed using the tree of Figure 2 EaCh Line Corresponds to a SEED LETTER (AMINO ACID PARTITION) 
to the protocol described in Section $\mathrm{V}-\mathrm{B}$. Each seed is then associated with a point on a unit square with coordinates corresponding to sensitivity and selectivity (see plots in Figure 6 below). The distance of this point to point $(1,1)$, denoted $\rho(\alpha)$, measures how good the sensitivity and selectivity jointly are. Besides, the number of occurrences of $\alpha$ in the seed should be taken into account. Overall, we chose to compute the score of a letter by the following formula:

$$
w(\alpha)=\sum_{\pi} \operatorname{occ}_{\pi}(\alpha) \cdot(\sqrt{2}-\rho(\alpha)),
$$

where the sum is taken over all seeds $\pi$ of a given span, and $\operatorname{occ}_{\pi}(\alpha)$ is the number of occurrences of $\alpha$ in $\pi$.

Greedy algorithm. Once every seed letter has been assigned a score, we compute the greedy solution as follows. We compute the maximal subset $L$ of locally good letters, i.e. letters $\alpha$ that score better than any letter $\alpha^{\prime}$ such that $\left\{\alpha, \alpha^{\prime}\right\} \notin \mathcal{I}$. It can be shown that this subset is independent and is included in the solution computed by the greedy algorithm. Then we redefine $E$ and $\mathcal{I}$ by $E^{\prime}=E \backslash L$ and $\mathcal{I}^{\prime}=\left\{Z \subseteq E^{\prime} \mid Z \cup L \in \mathcal{I}\right\}$ and apply the algorithm recursively to the independence system $\left(E^{\prime}, \mathcal{I}^{\prime}\right)$. The union of all sets $L$ of locally good letters computed along this procedure forms the solution of the greedy algorithm.

Resulting alphabet. Figure 3 shows alphabet Transitive-predefined designed through the approach of this Section. The alphabet has been designed from the tree of Figure 2 and using the alphabet $\mathcal{B}_{\alpha}=\{-, \alpha\}$ for assigning the score of a letter $\alpha$. Each line in Figure 2 corresponds to a letter (amino acid partition). Among alphabets obtained by varying different parameters in scoring individual letters (such as the alphabet and seed spans used in the scoring procedure), alphabet Transitive-predefined produced best seeds and will be used in the experimental part of this work (Section $\mathrm{V}$ ).

\section{B. Transitive alphabets using an ab initio clustering method}

Hierarchical clustering of amino acids. A prerequisite to the approach of Section IV-A is a given tree describing a hierarchical clustering of amino acid based on some similarity measure. In this section, we describe an approach that constructs ab initio a hierarchical clustering of amino acids, using a likelihood measure. The approach can be seen as constructing a hierarchy of connected components of a graph based on the likelihood relation considered in Section III (see Figure 11) trying to build components with high likelihood values.

As in Section IV-A, our goal here is to construct a family of seed letters verifying the hierarchy condition (2). This family will be obtained with a simple greedy neighbor-joining 
clustering algorithm. We start with the partition of amino acids into 20 singletons. This partition corresponds to the \# letter. For a current partition $P=\left\{C_{1}, \ldots, C_{n}\right\}$, iteratively apply the following procedure.

1 For each pair of sets $C_{k}, C_{\ell}$,

1.1 consider the set $\operatorname{Bridge}\left(C_{k}, C_{\ell}\right)=\left\{\left(a_{i}, a_{j}\right) \mid a_{i} \in C_{k}, a_{j} \in C_{\ell}\right\}$.

1.2 compute ForeBridgeProb $(k, \ell)=\sum\left\{f_{i j} \mid a_{i} \in C_{k}, a_{j} \in C_{\ell}\right\}$ and

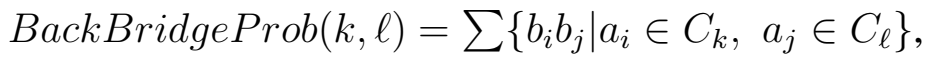

1.3 compute $L(k, \ell)=$ ForeBridgeProb $(k, \ell) / B a c k B r i d g e \operatorname{Prob}(k, \ell)$

2 Find the pair of sets $\left(C_{k}, C_{\ell}\right)$ yielding the maximal $L(k, \ell)$,

3 Merge $C_{k}$ and $C_{\ell}$ into a new set, obtaining a new partition.

The rationale behind this simple procedure is that those two sets of amino acids are merged together which produce the maximal increment in the likelihood. An alternative method, when the likelihood of the whole resulting set is maximized, yields biased results, as sets with a high likelihood tend to "absorb" other sets.

Resulting alphabet. An alphabet, called Transitive-ab-initio, obtained with this greedy neighbor-joining approach is given in Figure 4. It will be used in experiments presented later in Section $\mathrm{V}$.

\section{Non-hierarchical alphabets}

Previous approaches (Sections 4.1 and 4.2) were based on requirement (2) specifying that letters of the seed alphabet should be embedded one into another to form a "nested" hierarchy. This requirement is biologically motivated and, on the other hand, computationally useful as it reduces considerably the space of possible letters. However, this requirement is not necessary to implement the direct indexing (see Introduction). Therefore, we also designed non-hierarchical alphabets in order to compare them to hierarchical ones.

To design non-hierarchical alphabets, we used a heuristic generalizing the one of Section IV-B. The heuristic consists of two stages: first, generate a big number (several thousands) of "reasonable" candidate letters, and then select from them an alphabet containing $\sim 20$ transitive letters (not necessarily forming a hierarchy).

The algorithm of the first stage exploits the standard paradigm of genetic algorithms: it consequently creates "generations" of transitive letters. The initial population consists of a single "identity" seed letter. At the $k$-th iteration $(k=1, \ldots, 19)$, each letter generates $p$ descendants, each having $(20-k)$ sets. 


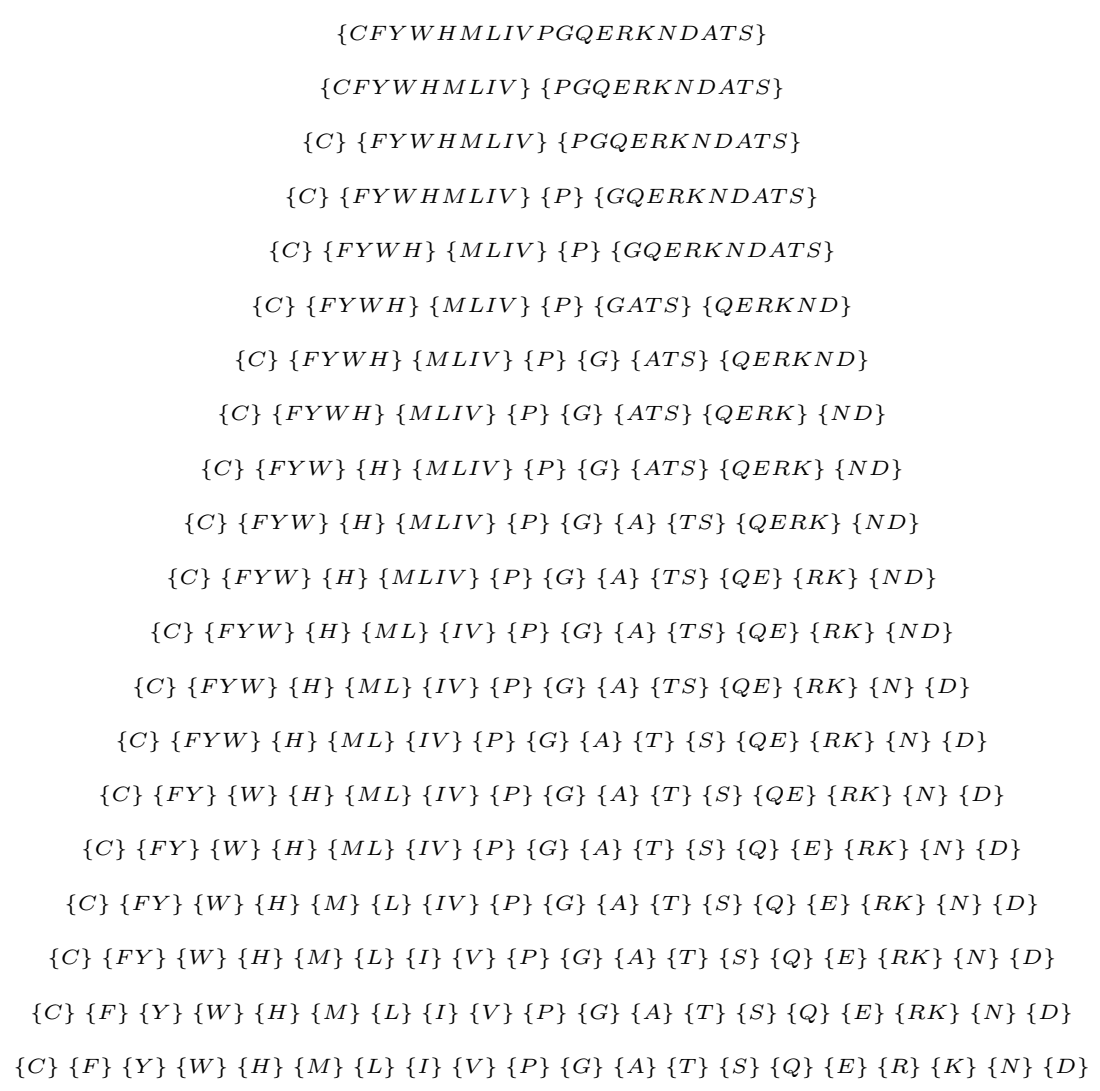

Fig. 4

Alphabet Trans itive-AB-initio obTained with the Method of Section IV-B

To generate descendants of a letter from the $k$-th generation, we use the algorithm given in Section IV-B but maintain $p$ (instead of just one) best partitions according to the likelihood of the "bridge". The $(k+1)$-th generation is selected among all descendants of the $k$-th generation by selecting those $q$ letters $\alpha$ which have the highest likelihood ratio $f(\alpha) / b(\alpha)$. With $p=100$ and $q=500$ the procedure gives about 8000 candidate letters.

To select a small number of those letters to form an alphabet, we tried different heuristics based on the following two ideas: (1) letters with high likelihood ratio are preferred (2) alphabet letters should have a range of different weights. The second option produced a better alphabet.

Resulting alphabet. We selected twenty letters out of about 8000 candidates by partitioning the candidates into twenty groups according to their weight ranging from 0 to 1 with increment 
0.05, and by picking in each group the letter with maximal likelihood. An alphabet obtained with the above heuristic, called Non-tree-transitive, is shown in Figure 5. This alphabet will be used in the experiments reported in Section $\mathrm{V}$.

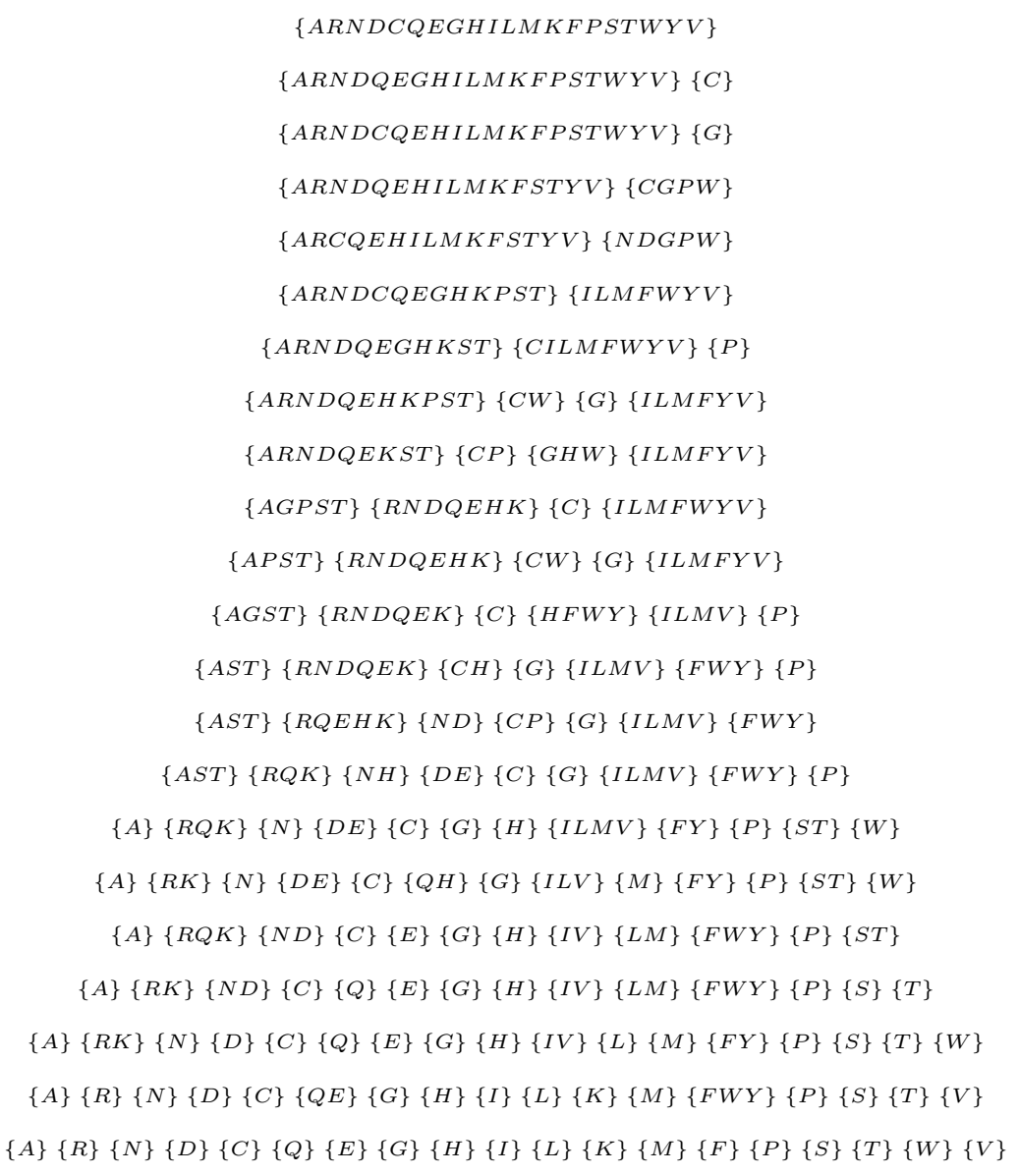

Fig. 5

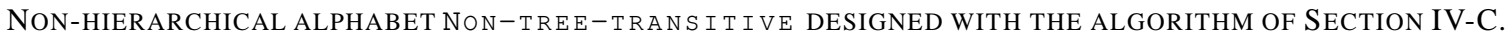

\section{EXPERIMENTS}

This section describes the experiments we made to test the efficiency of seeds we designed with different methods of previous sections. Sections $\mathrm{V}-\mathrm{A}-\mathrm{V}-\mathrm{C}$ describe the experimental protocol, from the assignment of background and foreground probabilities, to the seed design. In Section V-D, we analyze the power of different seed models proposed in 
Sections [II IV with respect to probabilistic models. Then in Section $\mathrm{V}$-E, we benchmark the performance of seeds built over different alphabets from Section IV against BLASTP, on several reference protein databases. For Sections $\mathrm{V}-\mathrm{D}$ and $\mathrm{V}-\mathrm{E}$, all relative experimental data including scripts, designed alphabets, seeds and seed families, and resulting sensitivity and selectivity measures, have been collected in a supplementary Web page available at http://bioinfo.lifl.fr/yass/iedera_proteins/,

\section{A. Probability assignment and alphabet generation}

First of all, we derived probabilistic models in accordance with the BLOSUM62 data from the original paper [26]. We obtained the BLOCKS database (version 5) [27] and the software of [26] to infer Bernoulli probabilities for the background and foreground alignment models. These probabilities have been used throughout the whole pipeline of experiments.

Different seed alphabets have then been generated by the methods presented in Section III (alphabet Non-transitive), SectionIV-A (alphabet Transitive-predefined), Section IV-B (alphabet Transitive-ab-initio) and Section IV-C (alphabet Non-tree-transitive).

\section{B. Seed design}

To each alphabet, we applied a seed design procedure that we briefly describe now. Since each seed (or seed family) is characterized by two parameters - sensitivity and selectivity - it can be associated with a point on a 2-dimensional plot. Best seeds are then defined to be those which belong to the Pareto set among all seeds, i.e. those than cannot be strictly improved by increasing sensitivity, selectivity, or both.

For different selectivity levels, we designed good seed families containing one to six individual seeds, among which the best family was selected. In each seed family, individual seeds have been chosen to have approximately the same weight, within $5 \%$ tolerance. This requirement is natural as in the case of divergent weights, seeds with lower weight would dominantly affect the performance. In practice, having individual seeds of similar weight allows an efficient parallel implementation (see e.g. [17]).

Estimation of sensitivity of individual seeds or seed families has been done with the algorithm described in [1] and implemented in the IEDERA software, available at http://bioinfo.lifl.fr/yas The selectivity of an individual seed has been computed according to the definition (Sec- 
tion (II). For a seed family, its selectivity has been lower-estimated by summing the background probabilities of individual seeds.

Seed family design has been done using a hill climbing heuristic (see [28], [29]) alternating seed generation and seed estimation steps. All experiments were conducted for alignment lengths 16 and 32 .

\section{BLASTP and the vector seed family from [4]}

Our goal is to compare between different seed design approaches proposed in this paper, but also to benchmark them against other reference seeding methods. We used two references: the BLASTP seeding method and the family of vector seeds proposed in [4]. Both of them use a score (or weight) resulting from the cumulative contribution of several neighboring positions to define a hit (see Introduction). Therefore, they use a more powerful (and also more costly to implement) formalism of seeding.

To estimate the sensitivity and selectivity of those seeds, we modified our methods described in the previous section by representing an alignment by a sequence of possible individual scores. Foreground and background probability of each score is easily computed from those for amino acid pairs. After that, sensitivity and selectivity is computed similarly to the previous case.

\section{Results on theoretical models}

We compare the performance of the different approaches by plotting ROC curves of Paretooptimal sets of seeds on the selectivity/sensitivity graph. The two plots in Figure 6 show the results for alignment length 16 and 32 respectively. Red and green polylines show the performance of BLASTP with word size 3 and the vector seed family from [4], for different score thresholds. The other curves show the performances of different seed alphabets from Sections [II IV] represented by the Pareto-optimal seeds (seed families) that we were able to construct over those alphabets. As mentioned earlier in Section $\nabla-B$, each time we selected the best seed family among those with different number of individual seeds. Typically (but not exclusively), points on the plots correspond to seed families with 4 to 6 seeds. Typically, the seed span ranges between 3 and 5 (respectively, 3 and 6) for alignment length 16 (respectively, 32). Seeds with larger span $(>4)$ tend to occur in seed families with larger number of seeds $(>3)$. 
We observe that seeds over the alphabet of Section 피 (dark blue curve) are comparable in performance with the vector seed family from [4] and clearly outperform seeds over other alphabets. This result is interesting in itself, although in many cases this alphabet is not practical due to its incompatibility with the transitivity condition.

As for the other alphabets, they roughly show a comparable performance among them. For the alignment length 16 , our seeds perform comparably to BLASTP, with a slightly better performance for high thresholds and a slightly worse performance for low thresholds. On the other hand, for alignments of length 32, our seeds clearly outperform BLASTP. Note that the non-hierarchical alphabet from Section IV-C does not bring much of improvement, which might indicate that lifting condition (3) does not bring much of additional power. This point, however, requires further investigation.

\section{E. Results on real data}

We made large-scale tests of our seeds on real data by applying them to several main databases of protein alignments. Those databases are BALIBASE (version 3) [30], HOMSTRAD [31], IRMBASE (version 1) [32], OXBENCH (version 1.3) [33], PFAM (release 22) [34], PREFAB (version 4) [35], and SMART (version 4) [36].

First, since all above databases except for OXBENCH contain multiple alignments, we extracted from each of them a dataset of pairwise alignments. For this, pairs of aligned sequences have been randomly extracted from multiple alignments and matching gaps removed. To avoid a bias induced by big (in terms of the number of sequences) multiple alignments, we selected a smaller fraction of pairwise alignments from big multiple alignments than from small ones: the number of selected alignments varied from order of $n^{2}$ for small alignments to $\sqrt{n}$ for big ones. The total number of alignment processed in our experiments varied from 640 (IRMBASE) to more than 250000 (PFAM).

For all those datasets, we identified alignments detected by the BLASTP seed for different score thresholds (word length 3, BLOSUM62 matrix, score threshold 10 to 13). On the other hand, for each BLASTP score threshold, we identified the closest seed family in the Pareto set (cf Section $\mathrm{V-B}$ ) with equivalent or greater selectivity. This has been done for each of the three transitive alphabets proposed in Section [V] Selected seeds can be found at the supplementary material Web page http://bioinfo.lifl.fr/yass/iedera_proteins/.

Results are shown on Figure 7 B Both methods detect a very high fraction of alignments of IRMBASE (all of them for thresholds 10 and 11). The poorest sensitivity is observed 
on SMART where alignments represent small sequences of proteins domains of the same family. A relatively weak sensitivity on PREFAB is due to its method of obtaining alignments which is based on structural information and, at the first step, "does not incorporate sequence similarity”. Finally, HOMSTRAD combines both structural information (using FUGUE [37]) and sequence information (using PSI-BLAST [3]) which explains a better performance of seed-based search in this case.

Comparing the performance of subset seeds vs. BLASTP, the former show clearly a better performance on BALIBASE, PREFAB and PFAM. On OXBENCH, HOMSTRAD and SMART, the obtained sensitivity is very close to that of BLASTP. Globally, subset seeds show a better performance for higher selectivity levels (greater thresholds).

\section{CONCLUSION}

In this paper, we studied the design of subset seeds for protein alignments, which is a very attractive seeding principle that does not use scores at the hitting stage of the alignment procedure. The design of efficient subset seeds subsumes a design of appropriate seed alphabets, i.e. sets of seed letters that seeds can be built from. In this paper, we studied several approaches to designing alphabets. In Section III, we considered the most general case when seed letters are only required to induce a symmetric binary relation on amino acids. In Section IV] we focused on transitive seed alphabets, where seed letters are required to induce an equivalence relation. In Section $I \mathrm{IV}-\mathrm{A}$ we proposed an alphabet construction based on pre-defined hierarchical clusterings of amino acids, while in Section IV-B, we considered a construction based on an ad hoc clustering of amino acids based on the likelihood ratio measure. Finally, in Section IV-C we lifted the requirement of hierarchical clustering and considered alphabets with possibly "incompatible" letters (in the sense of embedding of equivalence classes).

The main conclusion of our work is that although the subset seed model is less expressive than the method of cumulative score used in BLASTP, carefully designed subset seeds can reach the same or even a higher performance. To put it informally, the use of the cumulative score in defining a hit can, without loss of performance, be replaced by a careful distinction between different amino acid matches without using any scoring system. From a practical point of view, subset seeds can provide a more efficient implementation, especially for largescale protein comparisons, due to a much smaller number of accesses to the hash table. In particular, this can be very useful for parallel implementations or specialized hardware (see 
e.g. [17], [18]).

Interestingly, the BLAST team reported recently in [38] that they used a reduced amino acid alphabet in order to allow for longer seeds while still keeping the hash table of acceptable size. (Note also that this idea has recently been independently applied in [39], in a slightly different context.) This is done, however, by translating one of the sequences into a compressed alphabet and still using neighborhoods and a cumulative hit criterion. In this work, we demonstrated that instead of that, one can apply carefully designed subset seeds to avoid using neighborhoods and scoring systems at the seeding stage, without sacrificing the performance.

Note that the seed design heuristic sketched in Section $\mathrm{V}-\mathrm{B}$ does not guarantee to compute optimal seeds, and therefore our seeds could potentially be further improved by a more advanced design procedure, possibly bringing a further increase in performance. This is especially true for seeds of large weight (due to a bigger number of those), for which our seed design procedure could produce non-optimal seeds, thus explaining some "drop-offs" in high-selectivity parts of plots of Figure 6.

As far as further research is concerned, the question of efficient seed design remains an open issue. Improvements of the hill climbing heuristic used in this work are likely to be possible.

Finally, it would be very interesting to further study the relationship between optimal seeds and seed letters those seeds contain. In particular, it often appeared in our experiments that optimal seeds contained "non-optimal" seed letters. Understanding this phenomenon is an interesting theoretical question for further study.

Acknowledgments. Parts of this work have been done during visits to LIFL of Ewa Szczurek (June-August 2006), Anna Gambin and Sławomir Lasota (August 2006) and Mikhail Roytberg (October-December 2006). These visits were supported by the ECO-NET and Polonium programs of the French Ministry of Foreign Affairs. Laurent Noé was supported by the ANR project CoCoGen (BLAN07-1_185484). Mikhail Roytberg and Eugenia Furletova were supported by grants RFBR 06-04-49249 and 08-01-92496, and INTAS 05-10000008-8028. The authors thank Ivan Tsitovich for fruitful discussions of statistical questions related to this work, and Mathieu Giraud and Marta Girdea for commenting on the manuscript.

\section{REFERENCES}

[1] G. Kucherov, L. Noé, and M. Roytberg, "A unifying framework for seed sensitivity and its application to subset seeds," Journal of Bioinformatics and Computational Biology, vol. 4, no. 2, pp. 553-570, April 2006, preliminary version in WABI 2005. 
[2] S. Altschul, W. Gish, W. Miller, E. Myers, and D. Lipman, "Basic Local Alignment Search Tool," Journal of Molecular Biology, vol. 215, pp. 403-410, 1990.

[3] S. Altschul, T. Madden, A. Schäffer, J. Zhang, Z. Zhang, W. Miller, and D. Lipman, "Gapped BLAST and PSI-BLAST: a new generation of protein database search programs," Nucleic Acids Research, vol. 25, no. 17, pp. 3389-3402, 1997.

[4] D. Brown, "Optimizing multiple seed for protein homology search," IEEE/ACM Transactions on Computational Biology and Bioinformatics, vol. 2, no. 1, pp. 29-38, 2005, early version appeared in WABI 2004.

[5] B. Ma, J. Tromp, and M. Li, "PatternHunter: Faster and more sensitive homology search," Bioinformatics, vol. 18, no. 3, pp. 440-445, 2002.

[6] W. J. Kent, "BLAT-the BLAST-like alignment tool,” Genome Research, vol. 12, pp. 656-664, 2002.

[7] M. Li, B. Ma, D. Kisman, and J. Tromp, "PatternHunter II: Highly sensitive and fast homology search," Journal of Bioinformatics and Computational Biology, vol. 2, no. 3, pp. 417-439, 2004, earlier version in GIW 2003.

[8] L. Noé and G. Kucherov, "YASS: enhancing the sensitivity of DNA similarity search,” Nucleic Acid Research, vol. 33, pp. W540-W543, 2005.

[9] D. Mak, Y. Gelfand, and G. Benson, "Indel seeds for homology search," Bioinformatics, vol. 22, no. 14, pp. e341-e349, 2006.

[10] M. Csürös and B. Ma, "Rapid homology search with neighbor seeds," Algorithmica, vol. 48, no. 2, pp. 187-202, june 2007.

[11] B. Brejova, D. Brown, and T. Vinar, "Vector seeds: an extension to spaced seeds," Journal of Computer and System Sciences, vol. 70, no. 3, pp. 364-380, 2005.

[12] Y. Sun and J. Buhler, "Designing multiple simultaneous seeds for DNA similarity search," in Proceedings of the 8th Annual International Conference on Computational Molecular Biology (RECOMB04), San Diego (California). ACM Press, March 2004.

[13] G. Kucherov, L. Noé, and M. Roytberg, "Multi-seed lossless filtration," in Proceedings of the 15th Annual Combinatorial Pattern Matching Symposium (CPM), Istanbul (Turkey), ser. Lecture Notes in Computer Science, vol. 3109. Springer Verlag, July 2004, pp. 297-310.

[14] I.-H. Yang, S.-H. Wang, Y.-H. Chen, P.-H. Huang, L. Ye, X. Huang, and K.-M. Chao, "Efficient methods for generating optimal single and multiple spaced seeds," in Proceedings of the IEEE 4th Symposium on Bioinformatics and Bioengineering (BIBE 2004), May 19-21, 2004, Taichung (Taiwan). IEEE Computer Society Press, 2004, pp. $411-416$

[15] J. Xu, D. Brown, M. Li, and B. Ma, "Optimizing multiple spaced seeds for homology search," in Proceedings of the 15th Symposium on Combinatorial Pattern Matching, Istambul (Turkey), ser. Lecture Notes in Computer Science, vol. 3109. Springer, July 2004, pp. 47-58.

[16] D. Kisman, M. Li, B. Ma, and L. Wang, "tPatternHunter: gapped, fast and sensitive translated homology search," Bioinformatics, vol. 21, no. 4, pp. 542-544, 2005.

[17] P. Peterlongo, L. Noé, D. Lavenier, G. Georges, J. Jacques, G. Kucherov, and M. Giraud, "Protein similarity search with subset seeds on a dedicated reconfigurable hardware," in Proceedings of the 2nd Workshop on Parallel Computational Biology, Gdansk (Poland), ser. Lecture Notes in Computer Science, vol. 4967, 2007.

[18] V. H. Nguyen and D. Lavenier, "Speeding up subset seed algorithm for intensive protein sequence comparison," in Proceedings of the 6th IEEE International Conference on Research, Innovation \& Vision for the Future (RIVF 2008), 2008, pp. 57-63.

[19] L. Noé and G. Kucherov, "Improved hit criteria for DNA local alignment," BMC Bioinformatics, vol. 5, no. 149,14 October 2004. 
[20] L. Zhou, J. Stanton, and L. Florea, "Universal seeds for cDNA-to-genome comparison," BMC Bioinformatics, vol. 9, no. $36,2008$.

[21] B. Ma and H. Yao, "Seed optimization is no easier than optimal Golomb ruler design," in Proceedings of the 6th Asia Pacific Bioinformatics Conference (APBC), january 2008, pp. 133-144.

[22] U. Keich, M. Li, B. Ma, and J. Tromp, "On spaced seeds for similarity search,” Discrete Applied Mathematics, vol. 138, no. 3, pp. 253-263, 2004, preliminary version in 2002.

[23] T. Li, K. Fan, J. Wang, and W. Wang, "Reduction of protein sequence complexity by residue grouping," Journal of Protein Engineering, vol. 16, pp. 323-330, 2003.

[24] L. Murphy, A. Wallqvist, and R. Levy, "Simplified amino acid alphabets for protein fold recognition and implications for folding," Journal of Protein Engineering, vol. 13, pp. 149-152, 2000.

[25] S. Cheng and Y.-F. Xu, "Constrained independence system and triangulations of planar point sets," in Computing and Combinatorics, 1995, pp. 41-50.

[26] S. Henikoff and J. Henikoff, "Amino acid substitution matrices from protein blocks," Proc. Natl. Acad. Sci. USA, vol. 89, pp. 10915-10919, 1992.

[27] _ _ "Automated assembly of protein blocks for database searching." Nucleic Acids Res., vol. 19, no. 23, pp. 6565$6572,1991$.

[28] J. Buhler, U. Keich, and Y. Sun, "Designing seeds for similarity search in genomic DNA," in Proceedings of the 7th Annual International Conference on Computational Molecular Biology (RECOMB03), Berlin (Germany). ACM Press, April 2003, pp. 67-75.

[29] L. Ilie and S. Ilie, "Long spaced seeds for finding similarities between biological sequences," in Proceedings of the 2nd International Conference on Bioinformatics \& Computational Biology (BIOCOMP), 2007, pp. 3-8.

[30] A. Bahr, J. Thompson, J. Thierry, and O. Poch, "BAliBASE (Benchmark Alignment dataBASE): enhancements for repeats, transmembrane sequences and circular permutations," Nucleic Acids Research, vol. 29, no. 1, pp. 323-326, 2001.

[31] A. Stebbings and K. Mizuguchi, "HOMSTRAD: Recent developments of the homologous protein structure alignment database," Nucleic Acids Research, vol. 32, pp. D203-D207, 2004.

[32] A. R. Subramanian, J. Weyer-Menkhoff, M. Kaufmann, and B. Morgenstern, "DIALIGN-T: an improved algorithm for segment-based multiple sequence alignment," BMC Bioinformatics, vol. 6, no. 66, 2005.

[33] G. Raghava, S. Searle, P. Audley, J. Barber, and G. Barton, "OXBench: a benchmark for evaluation of protein multiple sequence alignment accuracy,” BMC Bioinformatics, vol. 4, no. 47, 2003.

[34] R. Finn, J. Mistry, B. Schuster-Bckler, S. Griffiths-Jones, V. Hollich, T. Lassmann, S. Moxon, M. Marshall, A. Khanna, R. Durbin, S. Eddy, E. Sonnhammer, and A. Bateman, "PFAM: clans, web tools and services," Nucleic Acids Research, vol. 34, pp. D247-D251, 2006.

[35] R. C. Edgar, "MUSCLE: multiple sequence alignment with high accuracy and high throughput," Nucleic Acids Research, vol. 32, no. 5, pp. 1792-1797, 2004.

[36] I. Letunic, R. Copley, B. Pils, S. Pinkert, J. Schultz, and P. Bork, "SMART 5: domains in the context of genomes and networks," Nucleic Acids Research, vol. 34, no. 1, pp. D257-D260, 2006.

[37] R. Nunez Miguel, J. Shi, and K. Mizuguchi, Protein Structure Prediction: Bioinformatic Approach. International University Line publishers, 2001, ch. Protein Fold Recognition and Comparative Modeling using HOMSTRAD, JOY and FUGUE, pp. 143-169.

[38] S. Shiryev, J. Papadopoulos, A. Schäffer, and R. Agarwala, "Improved BLAST searches using longer words for protein seeding," Bioinformatics, vol. 23, no. 21, pp. 2949-2951, 2007. 
[39] P. Peterlongo, L. Noé, D. Lavenier, N. V. H., G. Kucherov, and M. Giraud, "Optimal neighborhood indexing for protein similarity search,” BMC Bioinformatics, vol. 9:534, 2008. 
B62_L16
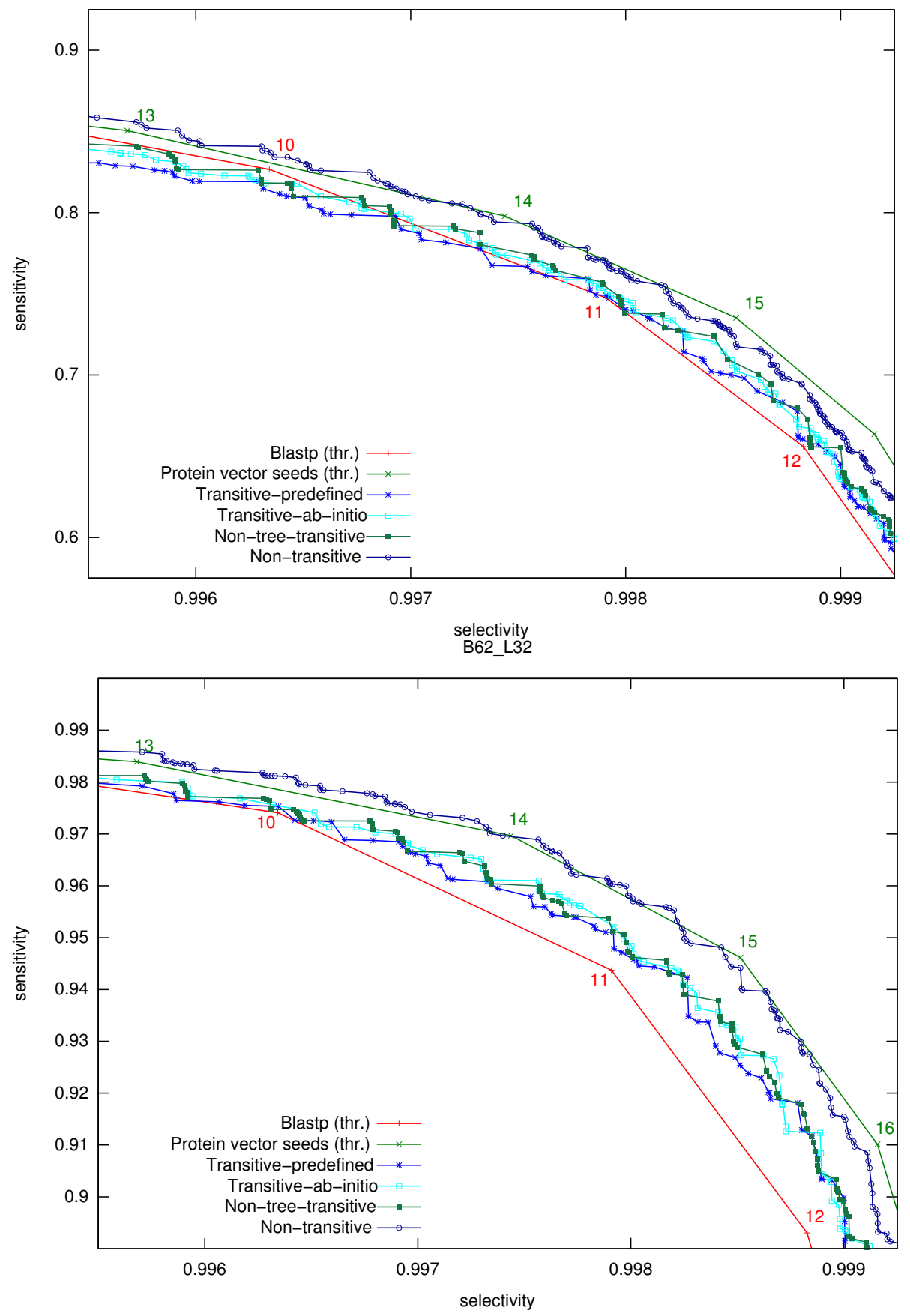

Fig. 6

ROC CURVES OF SEED PERFORMANCE MEASURED ON PROBABILISTIC MODELS FOR ALIGNMENT LENGTH 16 (ABOVE) AND 32 (BELOW). BLUE, CYAN DARK GREEN AND DARK BLEU CURVES REPRESENT PARETO-OPTIMAL SEED FAMILIES CONSTRUCTED RESPECTIVELY OVER ALPHABETS TRANSITIVE-PREDEFINED, TRANSITIVE-AB-INITIO, NON-TREE-TRANS ITIVE AND NON-TRANS ITIVE. EACH POINT OF THESE CURVES CORRESPONDS TO A SEED FAMILY, TYPICALLY 3 TO 5 SEEDS (RESPECTIVELY, 3 TO 6 SEEDS) FOR ALIGNMENT LENGTH 16 (RESPECTIVELY 32).

RED AND GREEN POLYLINES SHOW THE PERFORMANCE OF BLASTP WITH WORD SIZE 3 AND THE VECTOR SEED FAMILY FROM [4], FOR DIFFERENT SCORE THRESHOLDS. 

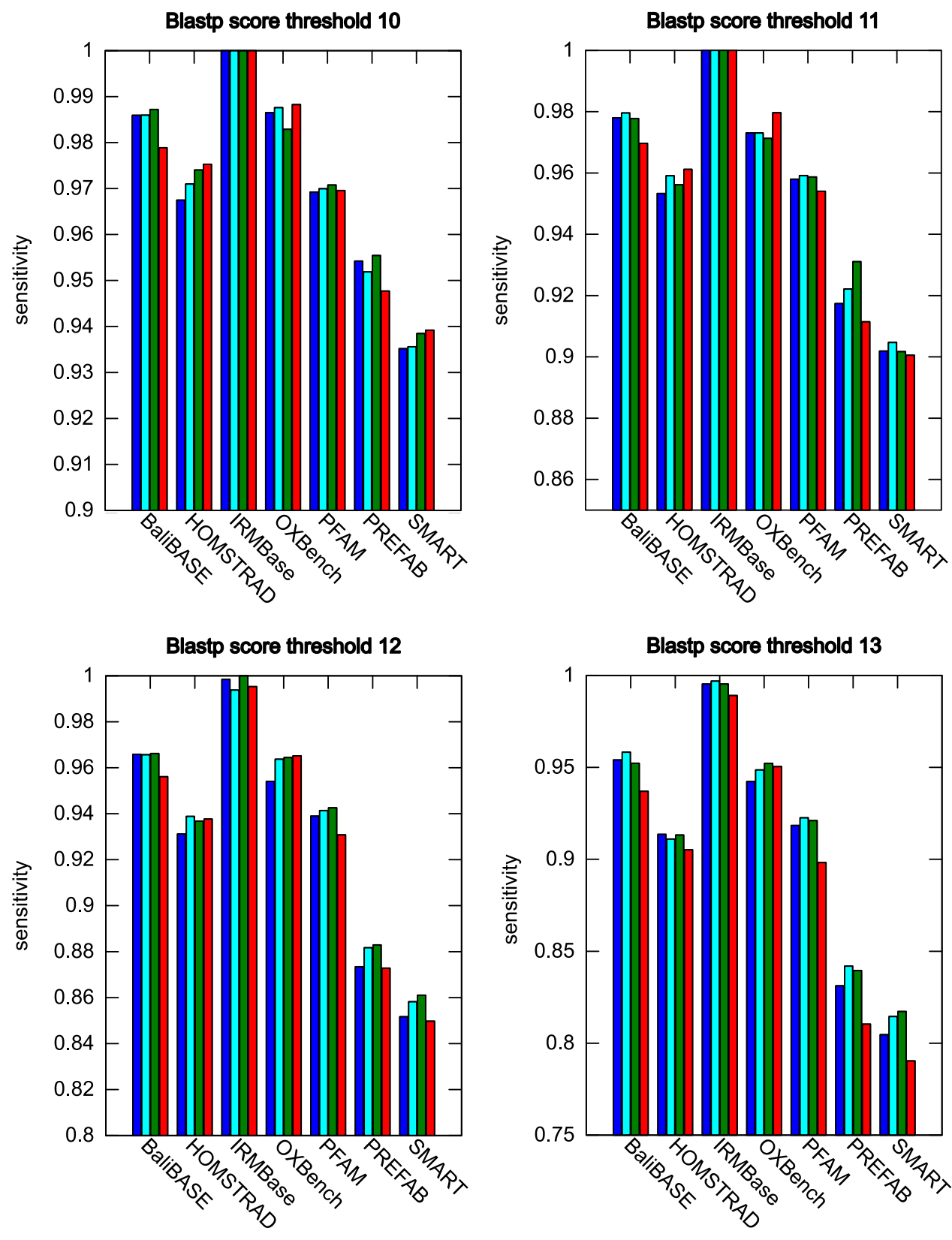

Transitive-predefined

Non-tree-transitive
Blastp

Fig. 7

SENSITIVITY OF SUBSET SEEDS VS BLASTP MEASURED ON BENCHMARK ALIGNMENT DATABASES 